\title{
MEASUREMENTS OF SOME ARGON PLASMA PARAMETERS GLOW DISCHARGE UNDER AXIAL MAGNETIC FIELD
}

\author{
Pshtiwan M.A. Karim ${ }^{\text {a }}$, Diyar S. Mayi ${ }^{b}$, Shamo Kh. Al-Hakary ${ }^{a}$ \\ ${ }^{a}$ Dept. of Physics, Faculty of Science, University of Zakho, Kurdistan Region, Iraq - (pshtiwan.karim, \\ shamo.awsi)@uoz.edu.krd \\ ${ }^{\mathrm{b}}$ Centre for material science and nanotechnology, Department of Physics, Faculty of Science, University of Zakho, Kurdistan \\ Region, Iraq (diyar.sadiq @uoz.edu.krd)
}

\begin{abstract}
:
This paper investigates the characteristics some of argon plasma parameters of glow discharge under axial magnetic field. The DC power supply of range (0-6000) $\mathrm{V}$ is used as a breakdown voltage to obtain the discharge of argon gas. The discharge voltagecurrent (V-I) characteristic curves and Paschen's curves as well as the electrical conductivity were studied with the presents of magnetic field confinement at different gas pressures. The magnetic field up to $25 \mathrm{mT}$ was obtained using four coils of radius 6 $\mathrm{cm}$ and 320 turn by passing A.C current up to 5 Amperes. Spectroscopic measurements are employed for purpose of estimating two main plasma parameters electron temperature $\left(T_{e}\right)$ and electron density $\left(n_{e}\right)$. Emission spectra from positive column (PC) zone of the discharge have been studies at different values of magnetic field and pressures at constant discharge currents of $1.5 \mathrm{~mA}$. Electron temperature $\left(\mathrm{T}_{\mathrm{e}}\right)$ and its density are calculated from the ratio of the intensity of two emission lines of the same lower energy levels. Experimental results show the abnormal glow region characteristics (positive resistance). Breakdown voltage versus pressure curves near the curves of paschen and decrease as magnetic field increases due to magnetic field confinement of plasma charged particles. Also the electrical conductivity increases due to enhancing magnetic field at different gas pressures. Both temperature density of electron and the intensities of two selected emission lines decrease with increasing pressure due decreasing of mean free path of electron. Electron density increase according to enhancing magnetic field, while the intensity of emitting lines tends to decrease.
\end{abstract}

KEYWORDS: Plasma Physics, Argon Gas, Paschen Law, Magnetic Field, CCS Spectrometer.

\section{INTRODUCTION}

Many kinds of material processing and surface modification applications can be carried out through the plasma of argon gas discharge. Since argon is a type of inert gas and has a convenient atomic mass amongst noble gases. The plasma diagnostics involves many techniques as electric probes and optical emission spectroscopy. But almost electric probes is favorite for measuring the parameters of plasma (Beck, 2000). Spectroscopic method is preferred as plasma diagnostic in case the plasma under the influence of magnetic field without the use of invasive probes that can perturb the behavior of the plasma. By using this technique one can calculates excitation, rotational, and vibrational temperatures, in addition to that temperature and density of others plasma species(Laux, 2001). Numerous empirical and theoretical works were carried out about with the presence of axial magnetic field upon the glow discharge at different argon pressures (Hassouba, 2001), (Makrinich, 2009), (Gao, 2017) and (Wais, 2011). Empirical works taking in to consideration the effect of experimental conditions (discharge current, voltage and magnetic field strength) upon the intensity of radiation emitted from the negative region of a direct- current glow discharge (Toma, 2005), (Michael, 2015), (Rózsa, 1995) and (Eizaldeen, 2012). A first attempt to use the cylindrical coaxial magnetron discharge (CCMD), obtained in air, as a light source to realize a high efficiency of conversion of electric power to visible light and to minimize the cost of the light source (Toma, Rusu, \& Dorohoi, 2008). The ion beam current in an end Hall-type ion source with a magnetic mirror field are investigated regarding magnetic gradient (Tang, 2007). The plasma parameters of a hollow cathode arc discharge (HCAD ), have been calculated by employing optical emission spectroscopy under magnetic field (Avaria, 2009). This is for nitrogen gas and used (200-1100) nm (Kolpaková, 2011). (Mondal, 2012), performed study of the discharge current (DC) current density in glow discharge (GD) in an axial magnetic field, also the shape of counter-rotating ion and electron fluids in imposing upon a negative glow discharge(NGD) under action of axial magnetic field. Also, (Galaly, 2014), studies the cathode fall thickness exerted by the magnetic field using two different methods for Helium plasma. Investigation of electron temperature and electron density of helium gas plasma at low pressures $(0.05,0.1$, $0.2,0.3) \mathrm{mBar}$, have been done under the action of both longitudinal and transversal magnetic fields (Munther, 2015). The effects of magnetic field strength (MFS) and the distance between the poles for argon discharge current (DC) electrical discharges on the Paschen's curves are studied for magnetic field strengths $(0,400,855)$ Gauss and distances $(2,4,6,8) \mathrm{cm}$ (Khalaf, 2016). (Ohno, 2019), have made a detailed description of discharge structure around the recombination front region (RFR).In the present work, investigation and characterization of argon glow discharge plasma exerted by axial magnetic is concerned. The principle aim is to explain the variation of plasma parameters due to the application of external axial magnetic field, and eventually gas pressure. Furthermore the present study describes experimentally the main features of DC hollow electrodes discharge in the positive region, with and without effect of axial magnetic field. As well as study this influence on the calculated temperature and density of electron from the ratio

* Corresponding author

This is an open access under a CC BY-NC-SA 4.0 license (https://creativecommons.org/licenses/by-nc-sa/4.0/) 
of two lines intensity method as well as from the Stark broadening of argon spectral line $(811 \mathrm{~nm})$.

\section{EXPERIMENTS EQUIPMENT}

The discharge chamber is a Pyrex glass of cylindrical shape. The chamber made as two parts, the first is $0.6 \mathrm{~cm}$ diameter and $14 \mathrm{~cm}$ length. The discharge chamber was provided with two inlets, one of the inlet is used as inlet gas to chamber and the another is used as outlet gas connected with rotary pump, both of $1.5 \mathrm{~cm}$ outer diameter and $7 \mathrm{~cm}$ in length. The second part is the electrodes chamber of $3 \mathrm{~cm}$ in diameter and of length $7 \mathrm{~cm}$ as illustrated in figure (1).The discharge pipe has two open ends are used to controlling the distance between two electrodes to obtain best uniform plasma at fixed distance $14 \mathrm{~cm}$. Both electrodes are fixed into the chamber by using two cylindrical rubber stoppers to prevent gas leakage. The electrodes are made of $0.5 \mathrm{~cm}$ in diameter and $9 \mathrm{~cm}$ in length of brass metal's hollow cylindrical shape to achieve the best discharge in terms of stability and density within the discharge tube. The system is evacuated to the required pressure about $1 \times 10^{-3}$ mbar using mechanical rotary vacuum pump. Pure argon gases were introduced into the system via a needle valve, also the pressure is measured by using Pirani gage. DC power supply of range (0-6000) volt is used and obtaining argon glow discharge for different pressure. The voltage - current $\left(\mathrm{V}_{\mathrm{d}}-\mathrm{I}_{\mathrm{d}}\right)$ characteristics was measured using a digital multi meter. Four coils of radius $6 \mathrm{~cm}$ and 320 turn each one to make one coil of length nearly $14 \mathrm{~cm}$ to produce magnetic field by passing A.C current of values (0 to 5) Amperes. The magnetic field obtained was ranged from (0 to 25) $\mathrm{mT}$ which is measured using Digital Gauss/Tesla Meter (Model: CYHT201 from Chen Yang Technologies), the hall probe is utilized to measure both direct and alternating magnetic field strength. The discharge tube was placed inside the magnetic field as shown in picture of the system (2). The experimental starts when the chamber was evacuated to an ultimate pressure and then feeding the argon into the chamber, allowing the pressure to reach 100 mbar before pumping the gas out to the desired operating pressure. This process of chamber flushing was repeated three times prior to each experiment to pump out the residual gases to ensure that the plasma-forming gas was pure argon. Once the desired pressure was reached, the applied voltage is increased gradually until inception of glow discharge. With each applied discharge voltage, the corresponding discharge current was recorded. Under different values of magnetic field strength (B), a range of discharge voltage and their corresponding currents were recorded. Two collecting lenses were arranged to collect and analyze radiation emitted from the discharge under experimental conditions through the optical fiber with CCS spectrometer, (500-1000) nm (Model: CCS175/M from Thorlabs) having grating of $830 \mathrm{Line} / \mathrm{mm}$, $800 \mathrm{~nm}$ optical resolution of $0.6 \mathrm{~nm}$ full width half maximum (FWHM). The optical fiber can be fixed in horizontal direction to the plasma chamber for characterizing and collecting radiation to determine the plasma parameters. For all experimental measurements when using spectrometer, calibration is necessary. This can be carried out using emission sources with known wavelengths (such as spectral lamps or helium-neon laser ) or for instance, one lines of the Balmer series belonging to a species present in the plasma (Menmuir, 2007).To be sure that the line intensities represents the corresponding excited level. There are three steps calibration process(Emission spectroscopy, Lab 57). First using standard spectral lamp within the selected spectral range in which strong peaks appear. In our experiment, we use a He-Ne gas laser as a standard lamp as shown in figure 3. Second identify the peak position along the $\mathrm{X}$-axis and then choose the maximum intensity of peaks in spectrum.
Third make comparison with the reference data taken from the handbook of the NIST Atomic Spectral Data (Kramida, NIST Atomic Spectra Database, 2019.).

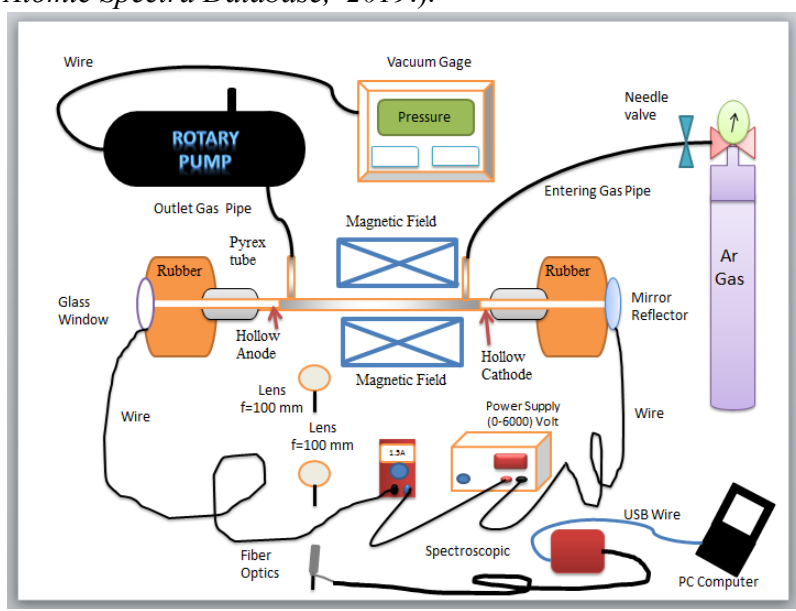

Figure 1. Schematic diagram of glow discharge with electric circuit.

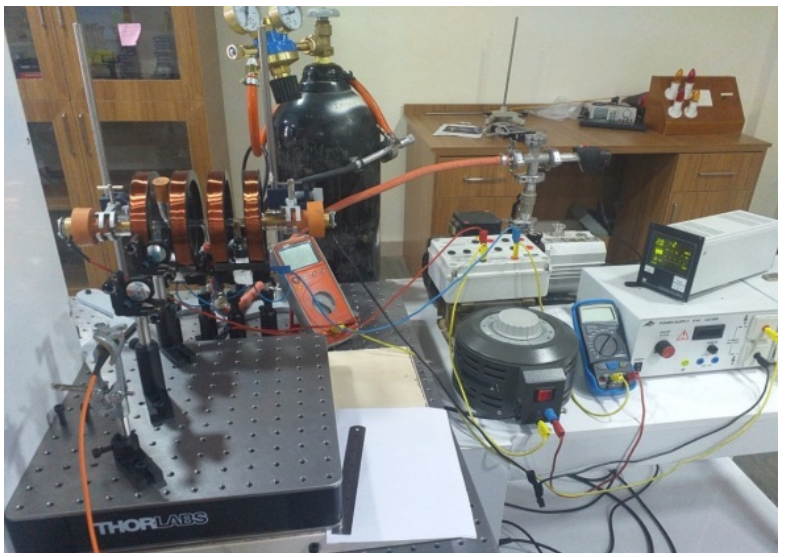

Figure 2. Picture of glow discharge system with magnetic field

\section{RESULTS AND DISCUSSION}

\subsection{Axial variation of magnetic field:}

The distribution of axial magnetic field through the discharge chamber was carried out when the Hall probe is employed in different positions between anode and cathode. The nearly uniform constant field strength (B) is obtained in horizontal direction of discharge tube as shown in figure (3).

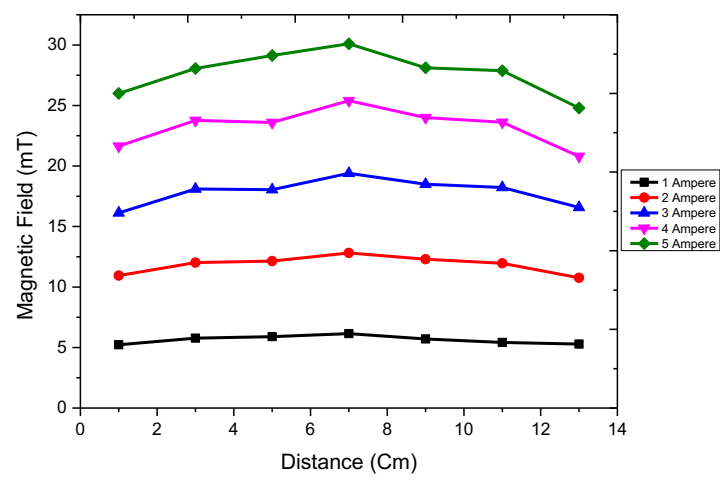

Figure 3. Variation of axial magnetic field through the discharge chamber for various positions.

3.2 The $\left(V_{d}-I_{d}\right)$ Characteristics of glow discharge 
The $\left(V_{d}-I_{d}\right)$ characteristics behavior of argon discharge obtained for pressure $(0.022-0.04)$ mbar, when the gap is 14 $\mathrm{cm}$ in length. These characteristics are represented abnormal glow region (positive resistance) as shown in figures (4). The remarkable behavior of these characteristics is the shifting to higher values of discharge voltage due to increasing the field. The discharge voltage under action of magnetic field for argon is larger than those without field. This effect was observed for low values of magnetic field. This mean that magnetic field increase the resistance of discharge for low values of magnetic fields ( 0 to 10$) \mathrm{mT}$, due to non - ionizing collision of electrons with other plasma particles (Baranov, 2011). For larger values of magnetic fields (15 to 25$) \mathrm{mT}$, Values of the discharge voltage when applying the field for argon are less than those in case the field is turned off for higher values of field. Thus, the slopes of the latter $\left(V_{d}-I_{a}\right)$ characteristics with the field are less than those without of field. This effect is attributed to that the field enhance the observed pressure and thus reducing mean free path of electron, therefore increasing the number of excitation and ionization collisions and due to that the resistance will be decreased as shown in figures (5).

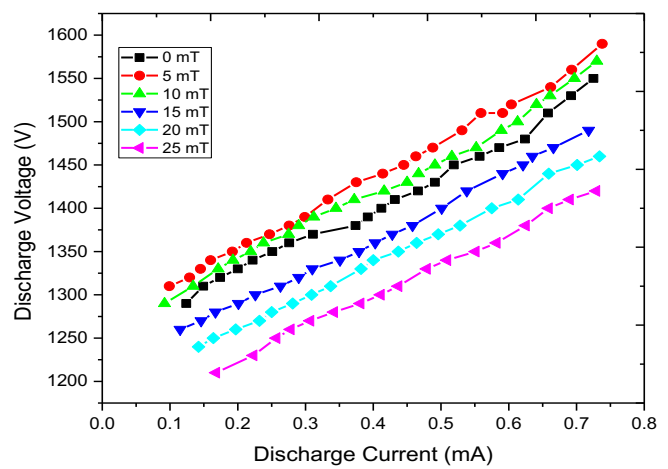

Figure 4. $\left(\mathrm{V}_{\mathrm{d}}-\mathrm{I}_{\mathrm{d}}\right)$ characteristics for different values of magnetic field under the pressure of $2.8 \times 10^{-2} \mathrm{mBar}$.

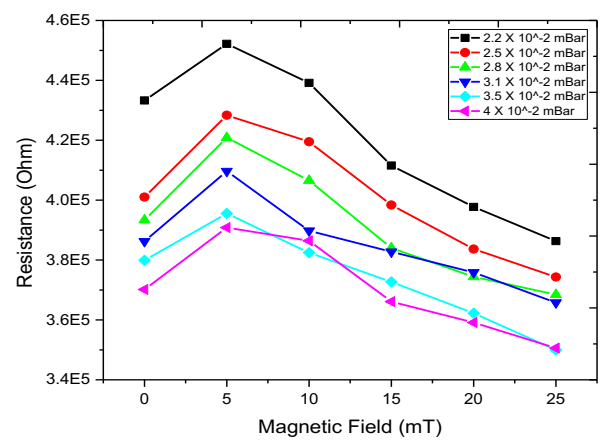

Figure 5. Electrical resistances versus axial magnetic field for several gas pressures.

By calculating the slope of $\left(\mathrm{V}_{\mathrm{d}}-\mathrm{I}_{\mathrm{d}}\right)$ characteristics in the abnormal region (positive resistance). For determinations the resistance and conductivity, (Origin Pro 9.0) software by linear fitting option for all (V- I) characteristic has been carried out. Electrical conductivity is a measure of how well a material accommodates the transport of the electric charge. According to Ohm's law the electrical conductivity $(\sigma)$ is given by the ratio of the current density $J$ in $\left(A / \mathrm{m}^{2}\right)$, to the electric field strength $E$ in $(V / m)$ (Haun, 2001):

$$
\sigma=\frac{J}{E}=\frac{L}{R A}
$$

Where $L, R$ and $A$ are the length of discharge in (m), discharge resistance in $(\Omega)$ and cross-section area in $\left(\mathrm{m}^{2}\right)$ respectively.
On the other hand, the electrical conductivity of non- ideal plasma is a fundamental quantity and its measurement, therefore, of high interest to verify new theories (Tkachenko, 1998). The discharge breakdown voltage $\left(\mathrm{V}_{\mathrm{b}}\right)$ is smaller under action of field, since the current density can be enhanced by the field, because the pressure will increase according to that. This effect is attributed to that the field enhance the observed pressure and thus reducing mean free path of electron, therefore increasing the number of excitation and ionization collisions and due to that breakdown voltage decreased as shown in Figure (6). On the other hand, the magnetic field increase the second ionization coefficient $\gamma$, at a certain value of reduce electric field $\mathrm{E} / \mathrm{P}$, in other words, both of the reduction of the work function of the cathode material since the application of the magnetic field is sufficient to maintain the discharge and magnetic field applied along the discharge axis promoted a reduction of the breakdown voltage agree with this reference (Petraconi, 2004).

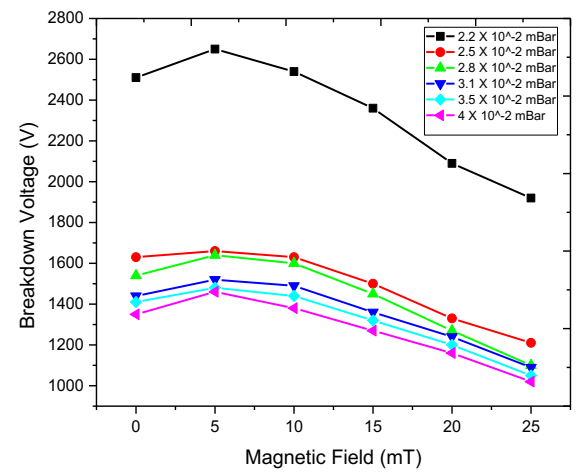

Figure 6 Breakdown voltages versus magnetic field for different values of gas pressure.

The pressure dependence of breakdown voltage in our study also has been taken into consideration. As well known the voltage breakdown is a function of the gas, gas pressure and electrodes gap separation ( Paschen's law), Friedrich Paschen developed a law in 1889, which is known as the Paschen's curve describing the breakdown voltage as a function of the electrode spacing or gap (d), operating pressure (p), and gas composition (Paschen, 1889) and (Torres, 2012). Therefore, the voltage breakdown $\left(\mathrm{V}_{\mathrm{b}}\right)$ versus gas pressure is plotted in figure (7). The results show similar behavior of Paschen's curves for different magnetic field.

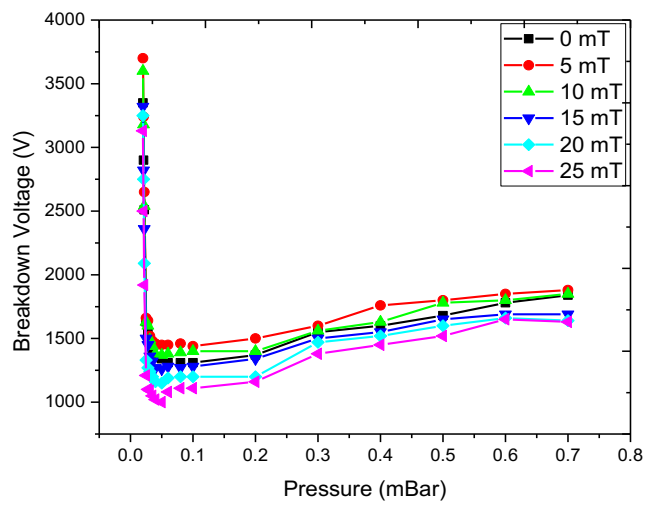

Figure 7 The breakdown voltage versus gas pressure for different values of the applied magnetic field.

\subsection{Electrical conductivity}

Figure (8), represents the increasing electrical of conductivity due to enhancing magnetic field at different gas pressures. The applying of the magnetic field prevents expansion of plasma species across the field. Measurement of the conductivity of the 
plasma is achieved directly by using general Ohm's law, i.e., calculating the slope of $\left(\mathrm{V}_{\mathrm{d}}-\mathrm{I}_{\mathrm{d}}\right)$ characteristics in the abnormal region ( positive resistance). According to the increase in the field, electron density and electrical conductivity are also increase. The motion of the electrons and other charged particles becomes finite in plane perpendicular to magnetic field, i.e. restricted the motion of the electrons and other charged particles therefore, leads to increase the density of the charged particles means increase the electrical conductivity. If the magnetic field is exerted axially to the discharge tube, this leads to a reduction of the breakdown voltage. This in turn makes magnetic confinement of electrons and ions which reduces the electron losses and effectively increases the collision frequency between electrons and the gas particles at a given $\mathrm{E}$ and $\mathrm{P}$ reduced field, consequently enhanced the rate of ionization efficiency. This means that the gas can be breakdown by lower ionization potential. In other word, they are decreasing the work function of the cathode material.

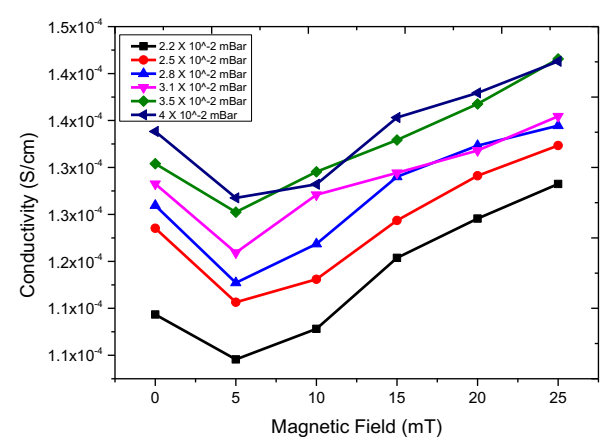

Figure 8 . The variation of electrical conductivity with a magnetic field for several values of gas pressures.

Also, it's noticeable from figure (9) that the electrical conductivity increase with increasing the pressure from (2.2 to $4 \times 10^{-2}$ ) mbar. Because these ranges of pressures is the left side of Paschen's curve were increasing pressure lead to increasing the number of excitation and ionization collision of electron with plasma particles, consequently decreasing the electrical resistance of discharge and breakdown voltage.

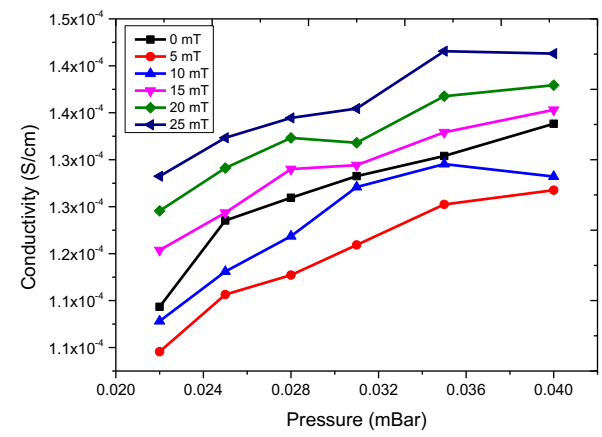

Figure 9. The electrical conductivity versus gas pressure for different values of the applied magnetic field.

\subsection{Spectroscopic Diagnostic:}

Spectroscopic method is a well-known and most popular technique for measuring some of the plasma parameters of glow discharges, because it is simple and don't perturbs the plasma. Electron temperature can be calculated from the ratio of two-lines emission of spectrum (Qayyum, 2003). In our study, the CCS spectrometer, $(500-1100) \mathrm{nm}$ is used for a wide survey spectrum of positive column region of argon glow discharge under external magnetic field. Figure 10, represents spectra obtained using an optical fiber, by collecting radiation emitted from the discharge employing two collection lenses of focal length (100) $\mathrm{mm}$ the first lens $(10) \mathrm{cm}$ far from the chamber wall, second lens about (10) $\mathrm{cm}$ far from first lens and the fiber optic about (10) $\mathrm{cm}$ far from second lens, which is the One of the best spectrums of argon discharge at pressure of $5 \times 10^{-2}$ mbar. Figure 2 explain that. The fiber output was connected to spectrophotometer of the following properties: optical resolution of $0.6 \mathrm{~nm}$. Spectral range (500-1100) nm, but only the emission lines between $(650-800) \mathrm{nm}$ were used to calculate electron temperature because the spectrum lines in this range are the best fitting with the National Institute of Standard and Technology (NIST) and these lines gives reasonable value of electrons less than 1 ev i.e. satisfy the local thermodynamic equilibrium (LTE) plasma. Argon emission spectrum at pressure $5 \times 10^{-2}$ mbar which is used to for temperature determination,

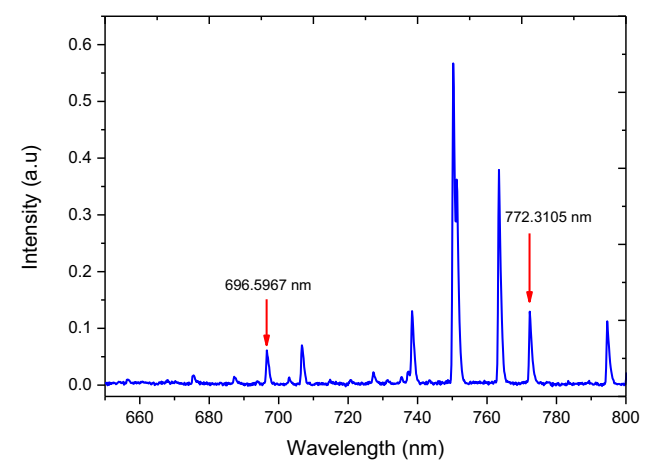

Figure10. The emission spectrum used for determination electron temperature under the pressure of $5 \times 10^{-2} \mathrm{mBar}$.

To show the spectroscopic parameters for spectral lines of Argon gas plasma in the current work shown in table 1 .

Table 1. Spectroscopic parameters for spectral lines identification of argon gas

\begin{tabular}{|c|c|c|c|c|c|}
\hline \multirow{2}{*}{ ions } & \multirow{2}{*}{$\begin{array}{c}\text { Wavelength } \\
\lambda(\mathrm{nm})\end{array}$} & \multirow{2}{*}{$\begin{array}{c}\text { Trans } \\
\text { ition } \\
\text { proba } \\
\text { bility } \\
A_{k} \\
\left(S^{-1}\right) \\
10^{6}\end{array}$} & \multicolumn{2}{|c|}{$\begin{array}{c}\text { Statistical } \\
\text { weight }\end{array}$} & \multirow{2}{*}{$\begin{array}{c}\text { Upper } \\
\text { energy } \\
\text { level } \\
(\mathrm{ev})\end{array}$} \\
\hline & & & $g_{i}$ & $g_{k}$ & \\
\hline ArI & 696.5967 & 6.4 & 5 & 3 & 13.32 \\
\hline ArI & 727.263 & 1.83 & 3 & 3 & 13.32 \\
\hline ArI & 772.3105 & 5.2 & 5 & 3 & 13.15 \\
\hline ArI & 811.434 & 33 & 5 & 7 & 13.07 \\
\hline ArI & 912.362 & 18.9 & 5 & 3 & 12.9 \\
\hline
\end{tabular}

To calculate the excitation temperature, line-to-line method was used (Danzaki, 2001). The Boltzmann plot can be used, according to equation (2) and equation (3) the spectral line intensity can be written as:

$$
E_{2}-E_{1}=h v_{12}
$$

Where $E_{1}$ and $E_{2}$ are the energies of levels 1 and 2, $h$ is the Planck constant and

$v_{12}$ is the transition frequency.

$$
I_{21}=\frac{N}{U(T)} g_{2} A_{21} h v_{21} e^{-E_{2} / K T}
$$

$U(T)$ is the average temperature of the electrons, $g$ is the statistical weight, $N$ is the population of level 1 and $2, I_{21}$ is the 
line intensity, $T$ is the electron temperature, $A_{21}$ is the transition probability of level 1 and 2, and $k$ is the Boltzmann constant.

By rewriting equation (3) as:

$$
I_{21}=\frac{N h c}{U} \frac{g_{2} A_{21}}{\lambda_{21}} e^{-E_{2} / k T}
$$

Where $c$ is the speed of light, $N, h, c$ and $U$ are the same for all of the atomic lines, therefore, and

$$
\begin{gathered}
\frac{U}{N h c}=k_{1}, \frac{I_{21} \lambda_{21}}{g_{2} A_{21}} k_{1}=e^{-E_{2} / k T}, \text { and } \\
\ln \left(\frac{I_{21} \lambda_{21}}{g_{2} A_{21}} k_{1}\right)=\ln \left(e^{-E_{2} / k T}\right)
\end{gathered}
$$

Where $\ln \left(\frac{I_{21} \lambda_{21}}{g_{2} A_{21}}\right)+k_{2}=-\frac{E_{2}}{k T_{e x}}$ where $k_{2}=\ln \left(k_{1}\right)$

By plotting $y=\ln \left(\frac{I_{21} \lambda_{21}}{g_{2} A_{21}}\right)$ versus $x=E_{2}$

A curve is obtained with a slope of $m=-\frac{1}{k T_{e x}}$ this method is widely used in the literature (Park, 2010). For obtaining a better understanding, two different transitions between energy states, $m-n$ and $p-n$, were considered; the first transition occurs between levels $m$ and $n$, where $m$ is the upper level and $n$ is the level with lower energy. The spectral line intensity is:

$$
I_{m n}=\frac{N}{Z} g_{m} A_{m n} h v_{m n} e^{-E_{m} / k T}
$$

The second transition is between energy levels $p$ and $n$, where $\mathrm{p}$ is the level with upper energy and $\mathrm{n}$ is the level with lower energy; the line intensity is given by:

$$
I_{p n}=\frac{N}{Z} g_{p} A_{p n} h v_{p n} e^{-E_{p} / k T}
$$

Where $(p>m)$ correlating these spectral lines, the next equation is obtained:

$$
\frac{I_{m n}}{I_{p n}}=\frac{g_{m} A_{m n} \lambda_{p}}{g_{p} A_{p n} \lambda_{m}} e^{-\left(E_{m}-E_{p}\right)} / k T
$$

Equation (10) can be rewrite as equation (11). Therefore, from equation (11) temperature of electron can be determined from the ratio of two-line emission spectrum equation (Forati, 2016).

$\frac{I_{1}}{I_{2}}=\frac{g_{1} A_{1} \lambda_{2}}{g_{2} A_{2} \lambda_{1}} \exp ^{\left[\left(-\frac{E_{1}-E_{2}}{K T}\right)\right]}$

Where $I_{1}$ and $I_{2}$ are the intensities of two line spectrum in arbitrary unit, $g_{1}$ and $g_{2}$ are the statistical weight, $\lambda_{1}$ and $\lambda_{2}$ are the wavelength of two line spectrum in (nm), $A_{1}$ and $A_{2}$ are the transition probability in $\left(\mathrm{S}^{-1}\right), E_{1}$ and $E_{2}$ are the upper energies level of two emission spectrum line in (ev), $k$ is the Boltzmann constant $=1.38064852 \times 10^{-23} \mathrm{~m}^{2} \mathrm{~kg} \mathrm{~s}^{-2} \mathrm{k}^{-1}$ and $T$ is the temperature in (Kelvin). The emission lines at $696.59 \mathrm{~nm}$ and $772.31 \mathrm{~nm}$ are chosen because they both of the same lower transition states (configuration $3 s^{2} 3 p^{5}\left(2 P_{3 / 2}^{0}\right)$ $4 \mathrm{~s}$, term ${ }^{2}[3 / 2]^{0}$ and $J=2$ ).

The upper states of the emission lines at $696.59 \mathrm{~nm}$ have the configuration $\left(3 s^{2} 3 p^{5}\left(2 P_{1 / 2}^{0}\right) 4 p\right)^{2}[1 / 2]$ and $J=1$, but for the emission line $772.31 \mathrm{~nm}$ have the upper state configuration $\left(3 s^{2} 3 p^{5}\left(2 P_{3 / 2}^{0}\right) 4 p\right)^{2}[3 / 2]$ and $J=1$. Other parameters of the two lines are taken from the national institute of standards and technology (NIST) as: $\mathrm{E}_{696.5}=106087.2598 \mathrm{~cm}^{-1}$, $\mathrm{E}_{772.3}=107496.4166 \mathrm{~cm}^{-1}, \mathrm{~g}_{696}=3\left(\mathrm{~s}^{-1}\right), \mathrm{A}_{696}=6.4 \times 10^{6}$, and $\mathrm{g}_{772}=3\left(s^{-1}\right), \mathrm{A}_{772}=5.2 \times 10^{6}$. The measured intensities ratio of the two lines, as shown in Figure 10, was $\mathrm{I}_{696.5} /$ $I_{772.3}=0.4738$. Then, using equation (4), the plasma temperature was obtained to be $\mathrm{T}=0.1651 \mathrm{eV}=1900 \mathrm{~K}$. Figure (11) represents the argon energy level diagram.

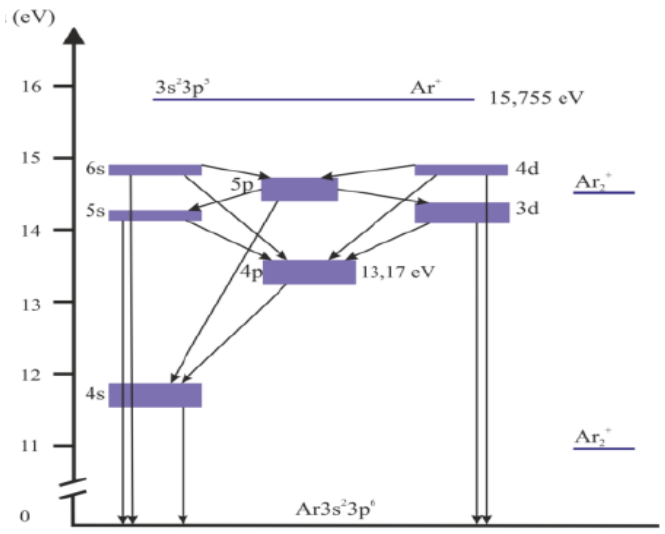

Figure 11. Argon energy level diagram taken from this reference (Marinova, 2015).

Density of electron can be obtained from the width of Stark broadening expression (Aragón, 2001), (Subedi, 2014) and (Sherbini,2012):

$$
\begin{aligned}
\Delta \lambda=2 w\left(n_{e} / 10^{16}\right) & \\
+ & 3.5 A\left(n_{e} / 10^{16}\right)^{5 / 4} \times\left(1-\frac{3}{4} N_{D}^{-1 / 3}\right) \\
& \times w
\end{aligned}
$$

Where $\Delta \lambda_{1 / 2}$ represent the FWHM of Stark broadened spectral peak, $w$ is the electron impact width parameter in $(\mathrm{nm}), N_{r}$ is the reference electron density; in the case of neutral atoms is $10^{16} \mathrm{~cm}^{-}$ ${ }^{3}$ (Qian, 2010), $A$ is the ion broadening parameter in (nm), $n_{e}$ is the electron density in $\left(\mathrm{cm}^{-3}\right)$ and $N_{D}$ is the number of particles in Debye sphere. The first term in equation (2) indicate the broadening due to the electron contribution and the second term to the ion broadening contribution. The ionic term is negligible. Then, equation (5) can be modified to simple form (Konjević, 2002):

$$
\Delta \lambda=2 w\left(n_{e} / 10^{16}\right)
$$

The line emission $(811 \mathrm{~nm})$ and its corresponding electron impact width parameter was obtained from (Luo, 2015). To be $\mathrm{w}=$ $0.0904 \mathrm{~nm}$. The full width at half maximum (FWHM) of this spectrum is shown in figure 12, was utilized after Lorentz curve fitting (Coons, 2011). The (FWHM) of the line is equal to $(811.98-811.11=0.86 \mathrm{~nm})$, this gives rise the electron density $n_{e}=0.86 \times 10^{16} / 0.1808 \mathrm{~nm}=4.805 \times 10^{16}\left[\mathrm{~cm}^{-3}\right]$.

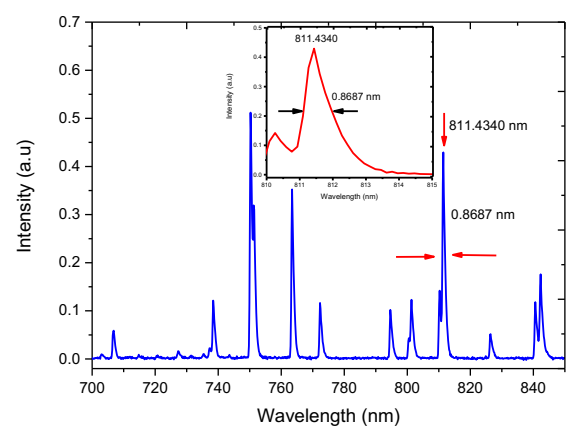

Figure

12 The emission line at $811 \mathrm{~nm}$ used for obtaining electron density.

The well resolved spectral lines of argon were used to extract the electron temperature $\left(T_{e}\right)$ and electron number density $\left(n_{e}\right)$. Electron temperature as well as electron number density are known to be independent of each other (Safeen, 2019). On the other hand the condition of high pressure plasma (HPP) but local 
thermodynamic equilibrium (LTE) must be satisfied for the validity of Boltzmann relationship. This condition allows finding the temperature varying from place to place. Generally, the plasma temperature was found by using two lines method. We observed that widths and intensities of identified spectral lines are directly related to magnetic field and both quantities are directly proportional to each other. The plasma temperature is calculated by changing the axial magnetic field from $(0-25) \mathrm{mT}$. The observed plasma temperature varies from about $1700-1900 \mathrm{~K}$ as shown in Figure13.

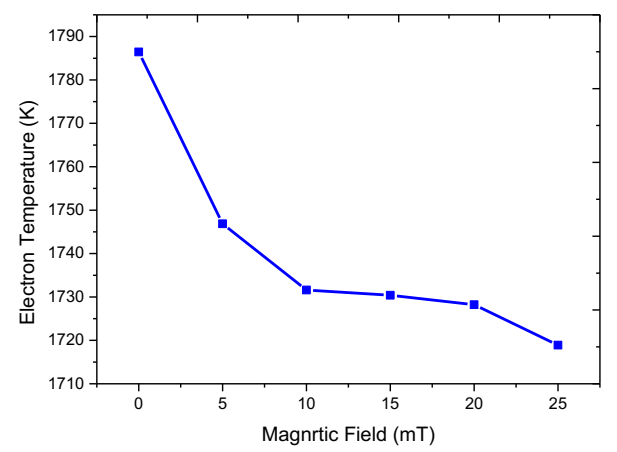

Figure 13 Electron temperature versus magnetic field under a $8 \times 10^{-2} \mathrm{mBar}$ pressure gas.

One of the important results here is that temperature of slow electrons (i.e., Maxwell-Boltzmann part) slightly decreases with enhancing magnetic field strength. While the fastelectron of high energy at the same time much more affected. Because the Electron paths are changed by a Lorentz force, this deflection is a function of the product of magnetic field intensity and electron velocity. Therefore, fast electrons are deflected and affected more than slow electrons. Hence will result in a higher number of excitations, ionizing and noncollisions for fast electrons (Boogaard, 2006). In other word, under axial magnetic field electrons move with helical path which increases the probability of collision with atoms and ions, this will reduce the effective free path and hence decreasing the velocity of the electrons and its energy. The results showed agreement with (Munther, 2015). The magnetic field dependence of electron density was plotted in figure 14, at different values of pressures.

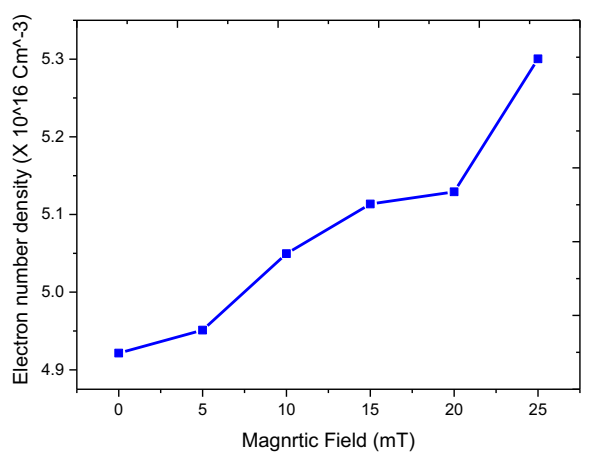

Figure14. Electron density versus axial magnetic field under a $3 \times 10^{-2} \mathrm{mBar}$ pressure.

When the plasma is subjected by an external axial magnetic field, the degree of ionization can be increased due to the confinement of the plasma charged particles. As a result of Lorentz force, the electron path will increases, leading to further frequent excitations and ionization collisions (Mohammed, 2017). Therefore electrons are moving through an axial magnetic field with helical paths around axial magnetic field due to magnetic confinement (Hassouba, 2001) and (Nahox, 2001), thus it may gain enough acceleration to ionize gas atoms leading to increasing electron density by inelastic collision with another particle (ion and atom helium). The most important result here is that for pressures $\left(3.5 \times 10^{-2}, 4 \times 10^{-2}, \ldots 5.5 \times 10^{-2}\right)$ mbar the electron density increase to saturation values approximately $5.5 \times 10^{16} \mathrm{~cm}^{-3}$ with enhancing magnetic field as shown in figure 15 .

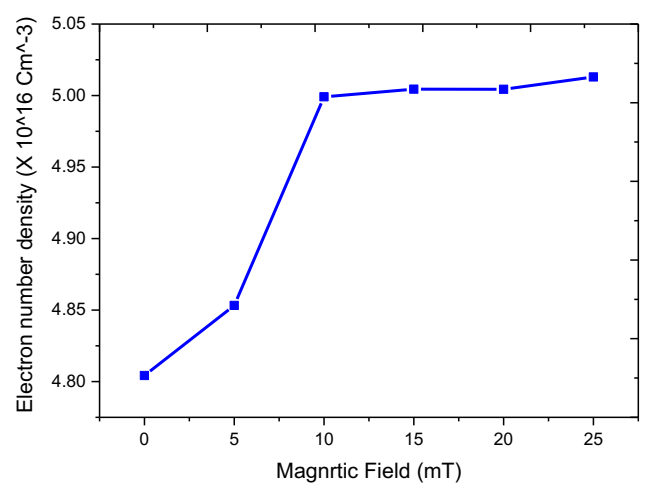

Figure 15 . Electron density versus magnetic field under a $5.5 \times 10^{-2} \mathrm{mBar}$ pressure.

It is obvious that the plasma under magnetic field is affected more noticeable at low pressure rather than high pressure, because at high pressure the mean free path of electron $\lambda_{\mathrm{e}}$ is very low and increasing magnetic will further reduce $\lambda_{\mathrm{e}}$. Consequently, the number of electron excitation collisions with other plasma charged particles is increased. Therefore, the electron will lose its energy and goes nearly too constant value as found in figure 15. Furthermore experimental result shows that the intensity of the lines emission decrease according to increasing axial magnetic field as illustrated from figure 16, because the applied axial magnetic field leads to increase the apparent gas pressure due to excitation and ionization collision of electron with plasma charged particle the high energy tail electrons (represented in energy distribution function are reduced (Boogaard, 2006). Consequently, the intensity of line transition will decrease.

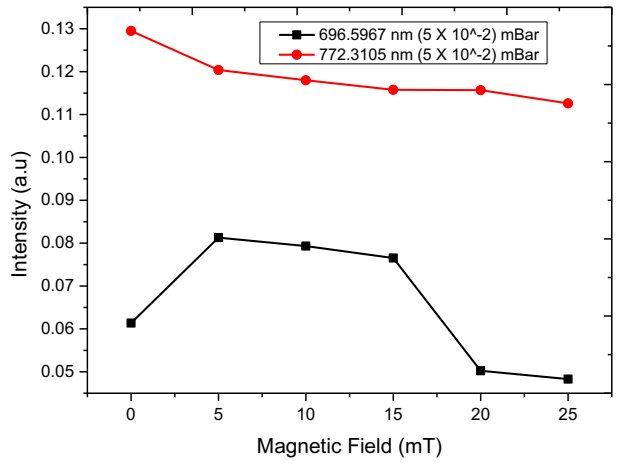

Figure 16. Intensity versus magnetic field.

It is appear that the two parameters are decreases approximately as an exponential function due to increasing gas pressure it is agreement with reference (Eizaldeen, 2012) as shown in figures 17 and 18. The variation is attributed to the fact that at low pressures, the electron temperature is much greater than the temperature of the gas, $T_{e} \geq T_{g}$. When the pressure in the plasma increases, the energy transfer from electron to the neutral gas, causing an increases in the temperature of the gas and decreases of the electron temperature. The results show good agreement with (KANEDA, 1978) and (Munther, 2015). 


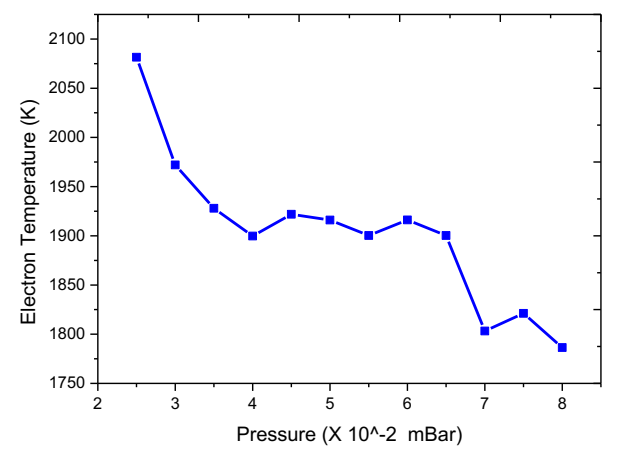

Figure 17. Electron temperature dependence of pressure.

Figure 18 illustrate nearly the decreasing exponential behavior of electron density versus pressure it can be interpreted in term of excitation and ionization collisions of atomic and ionic species in argon gas plasma. Enhancing gas pressure, the electron of high-energy tail (fast electron) represented in distribution function of electrons. The tail of the electron energy distribution function (which represents the high energy electron) shifted toward to the lower energies. Hence the ionization, due to these highly energetic electrons through collision with plasma particles, will decrease.

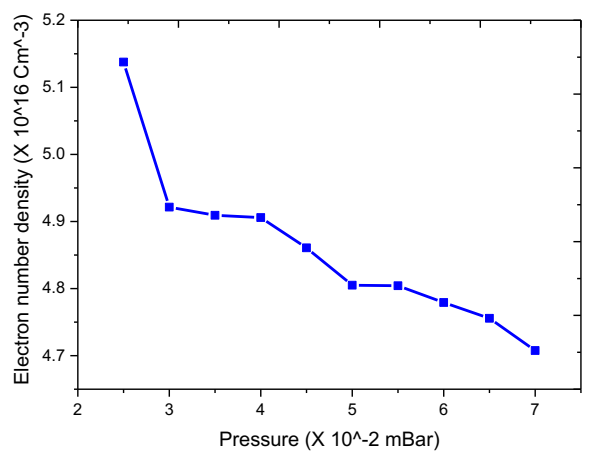

Figure 18. Variation of electron density with pressure.

Finally, another remarkable results is presented in figure 19, refer to gas pressure role on the emitted lines intensity shows that intensity of both chosen peaks $(696.59$ and 772.3$) \mathrm{nm}$ increase sharply from low pressure to medium pressure investigated and goes to nearly constant values of intensity due to further increasing of pressure. The behavior of the curve is indicated to the same reasons explained in reference(Qayyum, 2003).Therefore, the intensity of line transition due electron excitation collisions close to constant values.

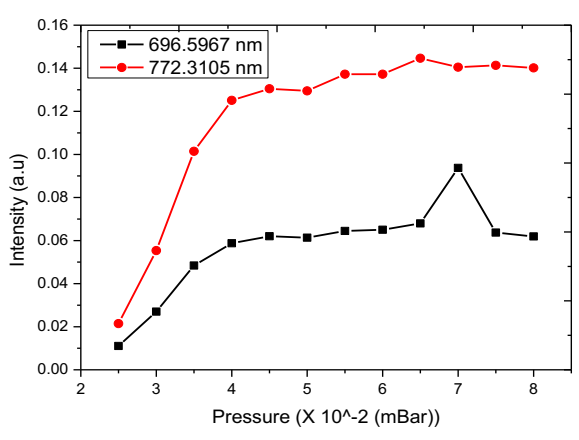

Figure 19. Variation of line intensity with pressure.

\section{CONCLUSION}

The argon glow discharge $\left(\mathrm{I}_{d}-\mathrm{V}_{\mathrm{d}}\right)$ characteristics obtained under several values of magnetic field and pressure represent abnormal region of glow discharge (positive resistance). These characteristics shifted downward to lower values of breakdown voltage due to increasing magnetic field. The latter effect is due to magnetic confinement to plasma charged particles. The electrical conductivity also increases according to both enhancing magnetic field and gas pressure from (2.3 to 3.5$) \times 10^{-2}$ mbar. This change in gas discharge characteristic can be attributed to the transition from the lower values of pressuredistance product of Paschen's curve to the high values of this product. The exert of axial magnetic upon (I-V) characteristic is equivalent to the effect of pressure, because as the applied axial magnetic field leads to increase the apparent pressure of the gas due to excitation and ionization collision of electron with plasma charged particle the high energy tail electrons (represented in energy distribution function are reduced. It was found from the experimental results that the two main argon plasma parameters are reduced by enhancing gas pressure. It was confirmed that the axial magnetic confinement has an effect to reduce the electron temperature, contrary to that increasing the electron density. Intensity of the selected lines decrease according to increasing axial magnetic field, while, the variation with pressure it's start to increase and goes to nearly constant values according to increasing gas pressure.

\section{REFERENCES}

Aragón, C., Bengoechea, J., \& Aguilera, J. A. (2001). Influence of the optical depth on spectral line emission from laser-induced plasmas. Spectrochimica Acta - Part B Atomic Spectroscopy, 56(6), 619-628. https://doi.org/10.1016/S0584-8547(01)00172-0

Avaria, G., Lunk, A., Schröder, A., \& Vinogradov, I. P. (2009). Optical and langmuir probe diagnostics in a magnetized hollow cathode arc. WILEY-VCH Verlag GmbH \& Co. KGaA, Weinheim, 6, S352-S356. https://doi.org/10.1002/ppap.200930804

Baranov, O., Romanov, M., Kumar, S., Zong, X. X., \& Ostrikov, K. (2011). Magnetic control of breakdown: Toward energy-efficient hollow-cathode magnetron discharges. Journal of Applied Physics, 109(6), 063304-063308. https://doi.org/10.1063/1.3553853

Beck, A., Hemmers, D., Kempkens, H., Schweer, H. B., \& Uhlenbusch, J. (2000). Comparative measurement of electron density and temperature profiles in low-temperature ECR discharges by a lithium atom beam and Thomson scattering. Journal of Physics D: $\quad$ Applied $\quad$ Physics, 33(4), 360-366. https://doi.org/10.1088/0022-3727/33/4/308

Boogaard, A., Kovalgin, A. Y., Aarnink, A. A. I., Wolters, R. A. M., \& Holleman, J. (2006). Measurement of electron temperatures of Argon Plasmas in a High-Density Inductively-Coupled Remote Plasma System by Langmuir Probe and Optical-Emission Spectroscopy. This Work Was Supported by the Dutch Technology Foundation (STW), under Project STW-TEL 6358, $412-418$.

C.O. Laux, R.J. Gessman, C.H. Kruger, F. Roux, F. Michaud, S. P. D. (2001). Rotational temperature measurements in air and nitrogen plasmas using the "rst negative system of N. Journal of Quantitative Spectroscopy and Radiative Transfer, 68(4), 473- 
482. https://doi.org/10.1016/s0022-4073(00)00083-2

Coons, R. W., Harilal, S. S., Polek, M., \& Hassanein, A. (2011). Spatial and temporal variations of electron temperatures and densities from EUV-emitting lithium plasmas. Analytical and Bioanalytical Chemistry, 400(10), 3239-3246. https://doi.org/10.1007/s00216-011-4792-y

Danzaki, Y., \& Wagatsuma, K. (2001). Effect of acid concentrations on the excitation temperature for vanadium ionic lines in inductively coupled plasma-optical emission spectrometry. Analytica Chimica Acta, 447(1-2), 171-177. https://doi.org/10.1016/S0003-2670(01)01261-2

Eizaldeen F. Kotp and Ashwaq A. AL-Ojeery. (2012). Studies The Effect Of Magnetic Field On Argon Plasma Characteristics. Australian Journal of Basic and Applied Sciences, 6(3), 817825.

Emission spectroscopy, Linkopings University, IFM - The Department of Physics, Chemistry and Biology, Lab 57. (n.d.).

Forati, E., Piltan, S., Li, A., \& Sievenpiper, D. (2016). Experimental study of the interaction between DC discharge microplasmas and CW lasers. Optical Society of America, 24(2), 14951506. https://doi.org/10.1364/oe.24.001495

Galaly, A. (2014). The Magnetized Plasma Effect on Cathode Fall Thickness for Helium Gas Discharge. Physical Science International Journal, 4(8), 1088-1099. https://doi.org/10.9734/psij/2014/9605

Gao, S., Chen, S., Ji, Z., Tian, W., \& Chen, J. (2017). DC Glow Discharge in Axial Magnetic Field at Low Pressures. Advances in Mathematical Physics, 9193149, 1-8. https://doi.org/10.1155/2017/9193149

Haun, J., Kosse, S., Kunze, H.-J., Schlanges, M., \& Redmer, R. (2001). Conductivity of Nonideal Zinc and Carbon Plasmas Experiments and Theoretical Results. Contributions to Plasma Physics, 41(2-3), 275-278. https://doi.org/10.1002/1521-3986(200103)41:2/3<275::aidctpp275>3.3.co;2-k

KANEDA, T. (1978). The influence ona of a transverse magnetic field glow discharge tube. J. Light \& Vis. Env., 2(1), 45-50.

Khalaf, M. K., Ali, D. S., \& Elttayef, A. K. (2016). Study the effect of longitudinal magnetic field and inter- electrode spacing on argon plasma discharges characteristics with Ti6Al4V alloy electrode. Al-Kut Univ. College Journal, 1(2), 2424-7419.

Kolpaková, A., Kudrna, P., \& Tichý, M. (2011). Study of Plasma System by OES ( Optical Emission Spectroscopy ). WDS'11 Proceedings of Contributed Papers, Part II, 180-185.

Konjević, N., Lesage, A., Fuhr, J. R., \& Wiese, W. L. (2002). Experimental Stark widths and shifts for spectral lines of neutral and ionized atoms (A critical review of selected data for the period 1989 through 2000). J. Phys. Chem. Ref. Data, 31(3), 819-927. https://doi.org/10.1063/1.1486456

Kramida, A., Ralchenko, Yu., Reader, J., and NIST ASD Team (2018). NIST Atomic Spectra Database (ver. 5.6.1), [Online]. Available: https://physics.nist.gov/asd [2019, February 8].
National Institute of Standards and Technology, Gaithersburg, $M D$. (n.d.). Retrieved from https://physics.nist.gov/cgibin/ASD/lines1.pl?spectra $=$ cu\&limits_type $=0 \& l o w \_w=320 \& u p$ p_w $=525 \&$ unit $=1 \& \mathrm{de}=0 \&$ format $=0 \&$ line_out $=0 \&$ en_unit $=0 \&$ o utput $=0 \&$ bibrefs $=1 \&$ page_size $=15 \&$ show_obs_wl $=1 \&$ show_cal c_wl $=1 \& u n c \_o u t=1 \&$ order_out $=0 \&$ max_low_enrg $=\&$ show_av $=$ 2\&max_upp_enrg $=\&$

Luo, W., Zhao, X., Lv, S., \& Zhu, H. (2015). Measurements of egg shell plasma parameters using laser-induced breakdown spectroscopy. Pramana - Journal of Physics, 85(1), 105-114. https://doi.org/10.1007/s12043-014-0893-4

M.A. Hassouba. (2001). Effect of the magnetic field on the plasma parameters in the cathode fall region of the DC-glow discharge. The European Physical Journal Applied Physics, 14(2), 131-135. https://doi.org/10.1051/epjap:2001148

Makrinich, G., \& Fruchtman, A. (2009). Experimental study of a radial plasma source. Physics of Plasmas, 16(4), 1-8. https://doi.org/10.1063/1.3119688

Marinova, P., Atanasova, M., \& Benova, E. (2015). Heavy particles and rate coefficients in $\mathrm{HF}$ and $\mathrm{MW}$ discharges in Argon at atmospheric pressure. 32nd ICPIG, Iași, Romania, 26(31), 31-33.

Menmuir, S. (2007). Visible spectroscopic diagnostics : Application and development in fusion plasmas (Doctoral Thesis Royal Institute of Technology Stockholm, Sweden). Retrieved from https://www.divaportal.org/smash/get/diva2:12758/FULLTEXT01.pdf

Michael A. Lieberman and Allan J. Lichtenberg. (2015). Principles of Plasma Discharges for Materials Processing. second edition, John Wiley \& Sons, Inc.

Mohammed, S. J., Khalaf, M. K., Majeed, M. A., \& Jasem, H. E. (2017). Experimental study on the effect of longitudinal magnetic field on Townsend discharge characteristics in low pressure argon gas. International Journal of ADVANCED AND APPLIED SCIENCES, 4(2), 91-95. https://doi.org/10.21833/ijaas.2017.02.016

Mondal, S., Narayanan, V., Ding, W. J., Lad, A. D., Hao, B., Ahmad, S., ... Kumar, G. R. (2012). Direct observation of turbulent magnetic fields in hot, dense laser produced plasmas. Proceedings of the National Academy of Sciences, 109(21), 8011-8015. https://doi.org/10.1073/pnas.1200753109

Munther B. Hassan and Hussain A. Hussein. (2015). The Effect of Magnetic Fields on Helium Plasma Parameters. JOURNAL OF KUFA - PHYSICS, 7(2), 35-39.

Nahox, I., Yoko, U., \& Nobuor, I. (2001). Effect of Magnetic-Mirror confinement on Electron Temperature Gontrol in ECR Plasma. $J$. Plasma Fusion Res., 4(3), 305-308.

Ohno, N., Seki, M., Ohshima, H., Tanaka, H., Kajita, S., Hayashi, Y., ... van der Meiden, H. (2019). Investigation of recombination front region in detached plasmas in a linear divertor plasma simulator. Nuclear Materials and Energy, 19, 458-462. https://doi.org/10.1016/j.nme.2019.03.010

Park, H., Choe, W., \& Yoo, S. J. (2010). Spatially resolved emission using a geometry-dependent system function and its application 
to excitation temperature profile measurement. Spectrochimica Acta - Part B Atomic Spectroscopy, 65(12), 1029-1032. https://doi.org/10.1016/j.sab.2010.11.008

Paschen, F. (1889). Ueber die zum Funkenübergang in Luft, Wasserstoff und Kohlensäure bei verschiedenen Drucken erforderliche Potentialdifferenz. Annalen Der Physik, 37, 69. https://doi.org/10.1002/andp.18892730505

Petraconi, G., Maciel, H. S., Pessoa, R. S., Murakami, G., Massi, M., Otani, C., ... Sismanoglu, B. N. (2004). Longitudinal magnetic field effect on the electrical breakdown in low pressure gases. Brazilian Journal of Physics, 34(4B), 16621666. https://doi.org/10.1590/s0103-97332004000800028

Qayyum, A., Ikram, M., Zakaullah, M., Waheed, A., Murtaza, G., Ahmad, R., ... Chaudhary, K. A. (2003). Characterization of Argon Plasma by Use of Optical Emission Spectroscopy and Langmuir Probe Measurements. International Journal of Modern Physics B, 17(14), 2749-2759. https://doi.org/10.1142/s0217979203018454

Qian, M., Ren, C., Wang, D., Zhang, J., \& Wei, G. (2010). Stark broadening measurement of the electron density in an atmospheric pressure argon plasma jet with double-power electrodes. Journal of Applied Physics, 107(6), 063303063305. https://doi.org/10.1063/1.3330717

Rózsa, K., Gallagher, A., \& Donkó, Z. (1995). Excitation of Ar lines in the cathode region of a dc discharge. Physical Review E, Vol. 52, pp. 913-918. https://doi.org/10.1103/PhysRevE.52.913

Safeen, A., Shah, W. H., Khan, R., Shakeel, A., Iqbal, Y., Asghar, G., .. Shah, W. H. (2019). Measurement of Plasma Parameters for Copper Using Laser Induced Breakdown Spectroscopy. Digest Journal of Nanomaterials and Biostructures, 14(1), 29-35.

Sherbini, A. M. El, Amer, A. A. S. Al, Hassan, A. T., \& Sherbini, T.
M. El. (2012). Spectrometric Measurement of Plasma Parameters Utilizing the Target Ambient Gas O I \&amp; N I Atomic Lines in LIBS Experiment. Optics and Photonics Journal, 02(04), 286293. https://doi.org/10.4236/opj.2012.24035

Subedi, D. P., Tyata, R. B., Shrestha, R., \& Wong, C. S. (2014). An experimental study of atmospheric pressure dielectric barrier discharge (DBD) in argon. Frontiers in Physics, AIP Conference Proceedings, 1588, 103-108. https://doi.org/10.1063/1.4867673

Tang, D., Zhao, J., Wang, L., Pu, S., Cheng, C., \& Chu, P. K. (2007). Effects of magnetic field gradient on ion beam current in cylindrical Hall ion source. Journal of Applied Physics, 102(12), 4-6. https://doi.org/10.1063/1.2825625

Tkachenko, I. M. (1998). Comment on "Thermodynamic and transport properties of dense hydrogen plasmas." Physical Review E Statistical Physics, Plasmas, Fluids, and Related Interdisciplinary Topics, 57(3), 3676-3677. https://doi.org/10.1103/PhysRevE.57.3676

Toma, M. (2005). Negative glow plasma as a converter of electric energy into radiation. Journal of Optoelectronics and Advanced Materials, 7(2), 687-690.

Toma, M., Rusu, I. A., \& Dorohoi, D. O. (2008). The influece of external magnetic field on the radiation emitted by negative glow of a DC glow discharge. Rom. Journ. Phys, 53(1-2), 295-301.

Torres, C., Reyes, G., Castillo, F., \& Martínez, H. (2012). Paschen law for argon glow discharge. Journal of Physics: Conference Series, 370(1), $\quad$ 012067-4. $\quad$ https://doi.org/10.1088/1742$6596 / 370 / 1 / 012067$

Wais, S. I., Mohammed, R. Y., \& Yousif, S. O. (2011). Influence of Axial Magnetic Field on the Electrical Breakdown and Secondary Electron Emission in Plane-Parallel Plasma Discharge. International Journal of Mathematical, Computational, Physical, Electrical and Computer Engineering, 5(8), 1226-1231. 